\title{
NÃO DEIXE AZEDAR: CONDICIONANTES DO DESPERDÍCIO ALIMENTAR À LUZ DE MARKETING SOCIAL
}

\author{
Stephanie Ingrid Souza Barboza ${ }^{1}$ \\ Flávio Perazzo Barbosa Mota ${ }^{2}$ \\ Erielem Araújo do Nascimento ${ }^{3}$
}

\section{RESUMO}

Este artigo analisou os fatores que influenciam o desperdício alimentar no nível doméstico a partir da definição de um modelo de hipóteses para predição da intenção a redução do desperdício alimentar. Pa ra o processo de coleta de dados, foi elaborado um questionário no Google forms, cujos respondentes fora $\mathrm{m}$ acessados através das redes sociais de grupos relacionados a alimentação, resultando em 532 questionários. Os dados foram submetidos a os procedimentos de validação psicométrica dos construtos, a análise das medidas descritivas e a análise das hipóteses do modelo proposto. Os resultados apontam que o grupo predisposto a redução do desperdício alimentar é composto por: mulheres, indivíduos entre $21 \mathrm{e}$ 35 anos, estudantes ou já graduados e que trabalham o dia inteiro. Tais resultados direcionam para a necessidade dos apelos de gênero além de outras possibilidades de segmentação dos programas de marketing social para redução do desperdício.

PALAVRAS-CHAVE: desperdício a limentar, marketing social, condicionantes, wicked problems

\section{DON'T LET IT SOUR: CONDITIONS OF FOOD WASTE IN THE LIGHT OF SOCIAL MARKETING}

\begin{abstract}
This article analyzed the factors that influence food waste at the household level from the definition of a hypothetical model to predict the intention to reduce food waste. For the data collection process, a questionnaire was created in Google forms, whose respondents were accessed through the social networks of groups related to food, resulting in 532 questionnaires. The data were submitted to psychometric validation procedures of the constructs, the analysis of descriptive measures and the analysis of the hypotheses of the proposed model. The results show that the group predisposed to reducing food waste is composed of: women, individuals between 21 and 35 years old, students or graduates and who work all day. These results point to the need for gender appeals in addition to other possibilities of segmenting social marketing programs to reduce waste.
\end{abstract}

KEYWORDS: food waste, social marketing, conditions, wicked problems.

\footnotetext{
${ }^{1}$ Universidade Federal da Paraíba,Paraíba (Brasil). https://orcid.org/0000-0002-2640-2070Email: stephanieisb@gmail.com

${ }^{2}$ Universidade Federal da Paraíba,Paraíba (Brasil). http://orcid.org/0000-0001-6812-1499 Email: fpmota@ gmail.com

${ }^{3}$ Universidade Federal da Paraíba, Paraíba (Brasil). http://orcid.org/0000-0001-7522-7478 Email: erielemadm@ gmail.com
} 


\section{INTRODUÇÃO}

O debate sobre desperdício alimentar tem emergido como uma forma de garantir bem-estar social a partir da redução da insegurança alimentar enfrentada por diversos países e dos problemas de saúde relacionados as questões alimentares, tais como a desnutrição. Em termos práticos, o desperdício alimentar é resultado da ineficiência do sistema alimentar em equilibrar o excesso e acesso das ofertas de alimentos, já que é comum pessoas descartando comida enquanto outros indivíduos passam fome (Närvänen, et al. 2019). Para a Organização das Nações Unidas para a Alimentação e Agricultura (FAO), a perda alimentar se refere à diminuição da massa disponível de alimentos para o consumo humano nas fases de produção, pós-colheita, armazenamento e transporte; já o desperdício está relacionado com as perdas derivadas da decisão de descartar alimentos que ainda têm valor e se associa, principalmente, ao comportamento dos vendedores, dos serviços de alimentação e dos consumidores finais (FAO, 2019).

Segundo Östergren et al. (2014), o desperdício de alimentos pode ser definido como qualquer alimento ou partes não comestíveis deles que são retirados da cad eia de suprimento para serem recuperados ou descartados. No entanto, o que tem sido classificado como desperdício é oriundo do exercício de diferenciação entre dimensões de evitabilidade versus inevitabilidade. Ou seja, o problema do desperdício de alimentos pode ser compreendido pelas características de desestruturação, transversalidade e implacabilidade, ao envolver um conjunto complexo e dinâmico de relações entre vários atores (sociedade, empresas e consumidores) e seus interesses (Närvänen, et al. 2020).

Närvänen, et al. (2020) compreendem o desperdício alimentar como um problema de difícil resolutividade, por envolver diversos agentes causando desequilíbrios no sistema alimentar, ao mesmo tempo em que são estes mesmos agentes que devem atuar nos diversos níveis para evitar o desajuste entre o que é demandado, consumido e descartado. Logo, é possível enquadra o desperdício alimentar como um wicked problem, uma vez que suas causas e efeitos são difíceis de identificar e não há uma definição clara do problema em si (Närvänen, et al. 2020).

É fato que o desperdício de alimentos envolve questões sociais, econômicas, nutricionais e ambientais, além de se configurar como desperdício de terra, água, energia e insumos (Halloran et al. 2014). Segundo a FAO (2019), o desperdício alimentar é responsável por gerar de $8 \%$ a $10 \%$ de todas as emissões de gases de efeito estufa produzidos por humanos, além de $38 \%$ dos recursos energéticos utilizados no sistema alimentar serem empregados na produção de alimentos que posteriormente serão perdidos ou desperdiçados. Como também, aproximadamente $30 \%$ das terras agrícolas do mundo são utilizadas na produção de alimentos que não serão consumid os. Em termos individuais, o brasileiro, por exemplo, desperdiça 353 gramas de comida por dia, representando $128,8 \mathrm{Kg}$ desperdiçados por ano.

Ou seja, o desperdício alimentar doméstico é reflexo da combinação entre elementos culturais de supervalorização da fartura e a valorização da comida fresca, conforme dito popular brasileiro "é melhor sobrar do que faltar", fortemente presente no contexto nacional (Porpino et al. 2018). Por outro lado, a redução do desperdício de alimentos pode afetar os custos dos alimentos, beneficiar o meio ambiente, melhorar a segurança alimentar e, prioritariamente, a saúde humana (Khalid et al., 2019; Benyam, Kinnear \& Rolfe, 2018). Porpino et al., (2018) reconhecem que o desperdício de alimentos está fortemente associado as questões comportamentais, em múltiplos momentos de consumo e incorporado a fatores contextuais e culturais.

Nesse contexto, a problemática do desperdício alimentar também passou a ser objeto de interesse do marketing social (Pellegrini et al., 2019; Stefan, 2013; Mirosa et 
al., 2016; Kim, Rundle-thiele \& Knox, 2019). Afinal, tal orientação, emergiu como a aplicação das tecnologias do marketing comercial para influenciar comportamentos voluntários em torno da promoção e do alcance de resultados que proporcionem o bemestar individual e social (Andreasen, 1994; Dibb, 2014). Classicamente, a aplicação do marketing social envolve a entrega de alternativas de comportamento que as pessoas valorizem e estejam dispostas a se sacrificar.

Dado que foco central das pesquisas de marketing social é o contexto da saúde, as discussões sobre questões que envolvam múltiplas dimensões de análise, tal como é o escopo do desperdício alimentar, ainda estão em fase de desenvolvimento tanto em termos práticos quanto teóricos. Em verdade, a maior parte das ações aplicadas no enfrentamento do desperdício são direcionadas a educar os indivíduos sobre características dos alimentos em termos de rotulagem, embalagem e conservação, as quais são executadas principalmente por meio de campanhas de informação e conscientização (Reisch et al., 2013). Entretanto, tais abordagens precisam ser aprimoradas, especialmente no que tange as práticas alimentares, transformando informações acerca dos impactos em ações cotidianas de redução do desperdício (Hebrok \& Heidenstrom, 2019). Ademais, Stefan et al., (2013) ainda reforçam que apesar dos consumidores serem os principais responsáveis pelo desperdício de alimentos nas residências, pouco se sabe a respeito dos fatores que levam a esse comportamento.

Dito isso, o presente estudo busca analisar os fatores que influenciam o desperdício alimentar no nível doméstico a partir da definição de um modelo de hipóteses para predição da intenção a redução do desperdício alimentar. O estudo compreende o desperdício alimentar como um wicked problem, exigind o a adoção de diversas perspectivas análise para auxiliar na ampliação do envolvimento do público e dos programas de marketing social, além das demais ações dirigidas ao combate do desperdício (Quested et al., 2013). A justificativa é que identificar fatores comportamentais pode contribuir para a promoção de ações mais focadas que busquem reduzir o desperdício alimentar. Para operacionalização da pesquisa, a seguir são apresentados: uma base teórica de marketing social, o conjunto de hipóteses de referência e os procedimentos metodológicos adotados. Por fim, são expostos os resultados e as considerações finais.

\section{MARKETING SOCIAL E OS CONDICIONANTES DO DESPERDÍCIO ALIMENTAR}

A área de marketing social se desenvolveu para atender demandas latentes da sociedade e com o objetivo de alcançar a maximização do bem-estar social, isto se deu através da construção de incentivos para a interrupção comportamentos existentes e muitas vezes arraigados na sociedade; da promoção para adoção de novos comportamentos desconhecidos, e, do convencimento das pessoas a modificar comportamentos existentes (Rundle-thiele et al., 2019). Além disso, especificamente na saúde, os programas de marketing social adotaram como estratégias: a venda de produtos subsidiados em pontos comerciais, a distribuição de produtos a preços adequados, o fornecimento de serviços de saúde por meio de franquias sociais e a promoção de comportamentos que não são necessariamente ligados a produtos e serviços (Firestone et al., 2017). 
A alimentação é um elemento central da saúde que tem sido abordada dentro do contexto de marketing social (Tobey et al., 2016; Ramirez et al., 2017; Saronghan, Thomson \& Ensor, 2016; Hodgkins et al., 2019). Como exemplo, pode ser citado o estudo que avaliou dois programas para melhorar as escolhas alimentares entre militares, sendo um programa voltado apenas para a comunicação direcionadas ao consumidor e outro com uma perspectiva de programa de marketing social que envolve todos os stakeholders envolvidos (Carins; Rudle-thiele \& Parkinson, 2017).

Apesar de muitas ações se concentrarem em tentar influenciar o comportamento do indivíduo, o marketing social também pode atuar na mudança de comportamento dos responsáveis por moldar os determinantes do comportamento humano, como os formuladores de políticas públicas (Dibb, 2014). Nesse contexto, o estudo de MonteroSimó e Araque-Padilla (2018) buscou entender a necessidade da ampliação do marketing social para além da mudança de comportamento individual, ao realizarem um estudo de caso sobre a “Jamie's Food Revolution”, uma campanha que envolveu diferentes agentes para pressionar o poder público a melhorar suas políticas de nutrição e alimentação. No que se refere ao nível individual, Worsley, Wang e Burton (2015) examinaram as opiniões dos consumidores a respeito das opções de políticas alimentares e a predisposição para comprar alimentos com viés pró-ambiental.

Em consonância com a perspectiva multinível, o caso do Banco Italiano de alimentos é um exemplo de estratégia de marketing social que usou a colaboração entre vários grupos de interesses envolvidos para criar um ambiente propício para mudança de comportamento de indivíduos, comunidades e a sociedade como um todo. A ação consistia na recuperação de alimentos que acabariam sendo descartados e na redistribuição para organizações de caridade, além de atuar na defesa de defesa de políticas públicas para a redução do desperdício alimentar (Silchenko, Simonetti \& Gistri, 2019; Santini \& Calvicchi, 2014).

É preciso ressaltar que nem toda intervenção na saúde pública, no contexto do desperdício, é baseada nos preceitos do marketing social. Chau et al, (2018) definiu um conjunto de pressupostos que orientam a identificação dos programas de marketing social. O primeiro deles envolve uma intervenção para a mudança de comportamento relacionado à saúde, na medida em que aborda conhecimentos, atitudes, crenças e outros antecedentes do comportamento a ser modificado. $\mathrm{O}$ segundo diz respeito a segmentação em grupos específicos, tendo por base as características demográficas, comportamentais e psicológicas. $\mathrm{O}$ terceiro trata da construção de um processo de troca, em que a intervenção oferta um valor social ajustado as demand as da sociedade e dos indivíduos objeto da mudança comportamental para justificar a adesão ao comportamento promovido. O quarto pressuposto se baseia na adoção das técnicas de marketing clássicas, como o design do plano de marketing social. Por fim, os autores reforçam a necessidade de a intervenção estar baseada em alguma teoria de mudança comportamental.

Com efeito, é importante que os programas, ações, iniciativas e políticas públicas que atuem na redução de desperdício de alimentos, considerem os resultados de estudos que analisem os fatores que condicionam a adoção de práticas alimentares sustentáveis. Nesse intento, foram analisados alguns trabalhos já publicados que integram os diversos fatores que influenciam o comportamento de desperdício alimentar, para então delimitar o conjunto de hipóteses apresentadas a seguir.

A princípio, Diaz-ruiz et al (2018) compreenderam que o desperdício alimentar pode ser considerado um hábito alimentar e está representado no estilo de vida adotado, na medida em que envolve as etapas de compra, armazenamento, preparação, consumo e descarte. Nos estudos realizados por Graham-Rowe et al., $(2014,2015)$ e Watson \& 
Meah (2012) foi observado que fatores internos ao indivíduo, como reações emocionais e convicções pessoais morais e éticas estavam diretamente relacionadas com o comportamento de desperdício alimentar. Em suas análises, os autores supracitados afirmam que o sentimento de obrigação moral sobre o desperdício de alimentos era frequentemente relatado nas entrevistas como uma motivação para desperdiçar menos comida.

No que diz respeito as convicções pessoais, Stancu, Haugaard \& Lähteenmäki (2015) e Visschers, Wickli \& Siegrist (2016) entendem que é preciso avaliar as normas que as pessoas adotam para comportamentos pró-ambientais, como é a adoção da redução do desperdício. Já no que se refere as reações emocionais, ressalta a relevância de analisar as emoções negativas e a culpa por desperdiçar alimentos como preditores do comportamento de redução. Sendo assim, são apresentadas as hipóteses H1 e H2.

H1. As convicções pessoais influenciam a predisposição para a redução do desperdício alimentar.

H2. As reações emocionais influenciam a predisposição para a redução do desperdício alimentar.

Por sua vez, certos hábitos de compra podem afetar o manejo familiar dos alimentos, como: o planejamento alimentar, a rotina de compras e as compras excessivas, conforme apresentados nos estudos de Porpino et al. (2015), Visschers; Wickli, \& Siegrist (2016) e Richter (2017). De fato, a prevenção ao desperdício de alimentos está ligada ao comportamento do consumidor (Quested et al., 2013), na medida em que ter uma rotina de compras se torna fundamental para limitar e orientar o sujeito na busca por alimentos (Stancu, Haugaard \& Lähteenmäki (2015). Por outro lado, comprar e cozinhar muita comida parece ser um dos principais propulsores do desperdício de alimentos (Brook Lyndhurst, 2007), uma vez que o planejamento de refeições com antecedência, a verificação de estoques e a reutilização das sobras, poderia contribuir para níveis mais baixos de desperdício de alimentos (Bell, Corsten, \& Knox, 2011; Visschers; Wickli, \& Siegrist, 2016).

Outra questão, é o fato da incompatibilidade do tempo entre as taxas e frequência de compra e o consumo de alimentos poder gerar a compra excessiva de alimentos e o aumento das chances de desperdício. Nesse caso, os consumidores que planejam melhor suas compras e refeições podem desperdiçar menos, se comparados aos consumidores que possuem uma alimentação baseada em improvisos desde a aquisição a preparação das refeições (Southerton \& Yates, 2015; Hebrok \& Heidenstrom, 2019). Nestes termos, são enunciadas as hipóteses H3, H4 e H5.

H3. A rotina de compras influencia a predisposição para a redução do desperdício alimentar.

H4. As habilidades domésticas influenciam a predisposição para a redução do desperdício alimentar.

H5. O planejamento alimentar influencia a predisposição para a redução do desperdício alimentar.

Parizeau et al. (2015) e Quested et al. (2013) identificaram uma avaliação negativa sobre o desperdício de alimentos, ao haver o senso de evitar desperdiçar e conservar recursos, já que partilhavam o sentimento de culpa após de jogar comida no lixo. Diaz-ruiz, Costa-font e Gil (2017) reconhecem que a capacidade do indivíduo de refletir sobre a geração de lixo alimentar pode ser um pressuposto para a adoção de um 
comportamento favorável à redução do desperdício. Além disso, a preocupação ambiental, ou seja, a consciência de que o desperdício de alimentos é também um desperdício de recursos ambientais, que gera lixo e impacta negativamente o meio ambiente, também pode influenciar a adoção de comportamentos favoráveis a redução do desperdício alimentar (Williams et al., 2018; Quested et al., 2013). Sendo assim, a hipótese H6 é anunciada.

H6. A gestão do lixo alimentar influencia a predisposição para a redução do desperdício alimentar.

Este conjunto de hipóteses sugere a atuação simultânea na formação da predisposição para a redução do desperdício alimentar. No intuito de testar essas hipóteses, foi então realizado um estudo empírico, sendo apontados no item seguinte os procedimentos metodológicos adotados.

\section{PROCEDIMENTOS METODOLÓGICOS}

No trabalho empírico foi desenvolvido um estudo por meio de procedimentos quantitativos, com base na coleta de dados primários. O uso de escalas de mensuração é sempre um desafio. Por isso, no que concerne aos elementos de mensuração, foram aplicadas escalas previamente testadas em estudos de interesse correspondente ao artigo. Isso permite, além de reduzir custos e tempo no processo de pesquisa, possibilidade de comparações interculturais (Gjersing et al., 2010). As escalas foram adaptadas para uma amplitude de 10 pontos (1-10; as aferições foram por meio de afirmações em escala de concordância do tipo Likert). Essa decisão tem como base o fato de que escalas de sete a onze pontos refletirem melhor a variação de uma população (Cox, 1980; Robinson et al., 1991), além de permitirem obter maior validade (Andrews, 1984).

Os construtos convicções pessoais e reações emocionais tiveram suas escalas oriundas das pesquisas de Visschers, Wickli \& Siegrist (2016) e Richter (2017). A escala de "rotinas de compras" foi extraída do estudo de Richter (2017). Para o construto "habilidades domésticas" utilizou-se as escalas advindas dos estudos de Stancu, Haugaard \& Lähteenmäki (2016) e Richter (2017). O construto planejamento alimentar teve suas escalas adaptadas do estudo de Richter (2017). Do estudo de Stancu, Haugaard \& Lähteenmäki (2016) foi utilizada a escala do construto gestão do lixo. Por fim, o construto predisposição foi adaptado dos estudos de Mondéjar-jiménez et al. (2016) e Stancu, Haugaard \& Lähteenmäki (2016). Feita a definição inicial das escalas do instrumento de pesquisa, um questionário foi construído, sendo acrescentadas ainda variáveis categóricas e demográficas para identificar o perfil dos respondentes.

Para o processo de coleta de dados, o procedimento de amostragem utilizado foi por acessibilidade e conveniência. Os dados foram coletados via internet, especificamente, por meio de um formulário online elaborado no Google Forms, disponibilizado através das redes sociais de grupos relacionados a alimentação, durante os meses de junho e julho de 2019. A coleta resultou em 532 questionários. Não houve eliminação de casos em decorrência dos procedimentos adotados na análise exploratória (vícios de respostas, valores duplicados ou perdidos e observações atípicas). A estratégia de compartilhamento em grupos de interesse do tópico de estudo é importante, algo indicado quando se trabalha com amostras por conveniência (Jager et al., 2017). Além disso, cabe ressaltar que mesmo com a dificuldade de se fazer 
generalizações nesse tipo de amostragem, ainda é possível se obter resultados não enviesados em modelos de regressão linear, desde que os pressupostos sobre os erros estimados sejam atendidos (Manion, 1994).

Dos 532 respondentes obteve-se que $53,9 \%$ são do sexo feminino e $46,1 \%$ são do sexo masculino. Quanto ao nível de escolaridade, 13,3\% possuem o ensino fundamental, 22,4\% concluíram o ensino médio, 44,4\% possuem curso superior e $19,9 \%$ possuem pós-graduação. No que concerne à idade dos respondentes, 55,8\% possuem entre 21 e 35 anos, 16,9\% tem idade inferior a 20 anos, 13,2\% estão entre 36 e 50 anos e 14,1\% informaram possuir mais de 51 anos. De acordo com os dados, 34,8\% dos respondentes possui renda familiar mensal entre $\mathrm{R} \$ 1.501,00$ e $\mathrm{R} \$ 4.000,00$ reais, $32,3 \%$ recebem até $R \$ 1.500,00$ reais mensais, $16,9 \%$ possuem a renda entre $R \$$ $4.001,00$ e 7.000,00 e os outros $16 \%$ dos respondentes informaram ter a renda mensal acima de R \$ 7.001,00 reais. Entre os respondentes, 34,4\% trabalham o dia inteiro, $15,4 \%$ trabalham meio período, 10,5\% informaram que são aposentados, $10,3 \%$ não estão trabalhando e $29,3 \%$ da amostra é composta por estudantes. Conforme dos respondentes assinalaram, $43 \%$ cozinham a própria refeição entre 2 e 4 dias por semana, $32,9 \%$ costumam fazer preparar suas refeições em casa mais de 5 dias por semana e $24,1 \%$ informaram cozinham a própria alimentação apenas 1 dia por semana.

O perfil demográfico apresentado indica a heterogeneidade pretendida para a amostra, assegurando boas condições para os procedimentos de análise empreendidos e minimiza as dificuldades, os potenciais problemas oriundos do procedimento de amostragem por acessibilidade. Os procedimentos estatísticos utilizados nesta pesquisa são os usualmente utilizados, quais sejam: análise das medidas descritivas, análise da consistência psicométrica dos construtos, comparação de diferenças de média entre grupos, análise correlação, modelo de hipóteses e conglomerados. Tais procedimentos foram realizados no software SPSS (SPSS Inc., Chicago, Estados Unidos), com base na literatura especializada (Lattin et al, 2011).

Para verificar a consistência psicométrica dos construtos, espera-se que, em cada construto, a variância extraída por meio da análise fatorial seja de pelo menos $50 \%$ e os escores fatoriais sejam superiores a 0,5 (Hair et al., 2005; Costa, 2011). Além disso, também se mensura a consistência interna por meio do Alpha de Cronbach, sendo esperado um valor mínimo de 0,6 (Costa, 2011). Para a análise do modelo de hipóteses, a técnica utilizada foi o modelo de regressão linear, a escolha foi pela estimação dos coeficientes pelos mínimos quadrados ordinários. As medidas de ajuste são verificadas em termos de estatística $F$ (com valor esperado do valor $p$ inferior a 0,05 ), coeficiente de determinação (quanto maior $\mathrm{o} \mathrm{R}^{2}$, melhor) e medidas de assimetria e curtose (com valores esperados entre -1 e 1) (Hair et al., 2005). Para os testes de comparação de diferenças de média entre os grupos, verifica-se se o valor da estatística t calculada para amostras independentes tem probabilidade inferior (valor $\mathrm{p}$ ) inferior a 0,05 e, para a análise de variância (ANOVA de um fator), verifica-se se o valor da estatística $F$ calculada tem probabilidade (valor p) inferior a 0,05 (Hair et al., 2005). Por fim, a análise de conglomerados foi realizada para tentar agrupar os respondentes em grupos com características comuns dentro dos grupos, mas com perfis diferentes entre os grupos. O método foi não hierárquico com formação de dois grupos pela distância mínima de cada centróide. A descrição dos procedimentos realizados é apresentada a partir do próximo tópico. 


\section{ANÁLISE DOS RESULTADOS}

Neste item, são apresentados os resultados do trabalho de campo. A princípio são expostos os procedimentos de validação psicométrica dos construtos, seguida da análise das medidas descritivas e, por último, a análise das hipóteses do modelo proposto.

\subsection{Validação psicométrica dos construtos}

Como indicado, cada construto foi medido por múltiplos itens que tinham relação supostamente refletiva em relação ao construto latente. Nestes termos, é necessário averiguar se o construto está bem mensurado do ponto de vista empírico, uma vez que a validação de conteúdo está aceita na escala de origem.

A averiguação ocorreu em duas etapas: inicialmente, foi realizada a análise fatorial, sendo esperado que em cada construto tenhamos uma variância extraída de ao menos 0,5 (50\%), e que os escores sejam de ao menos 0,5; em seguida, verificou-se a consistência interna por meio do Alpha de Cronbach, sendo esperado um valor mínimo de 0,6. Estas extrações foram procedidas construto a construto. Os resultados estão apresentados na Tabela 1 e indicam que os construtos foram mensurados adequadamente e todos apresentaram medidas de acordo com os valores desejáveis. Apenas para o construto "gestão do lixo" não foi calculada a confiabilidade, dado que o construto foi composto de apenas dois itens.

Tabela 1 - Resultados da análise psicométrica

\begin{tabular}{lcccc}
\hline \multicolumn{1}{c}{ Construtos } & $\mathbf{N}^{\circ}$ de itens & Var. extraída & Menor escore & Alpha \\
\hline Conviç̧ões pessoais (CP) & 4 & 66,413 & 0,750 & 0,829 \\
Reações emocionais (RE) & 3 & 85,407 & 0,902 & 0,915 \\
Rotina de compras (RC) & 5 & 54,566 & 0,663 & 0,790 \\
Planejamento de compras (PC) & 4 & 69,865 & 0,745 & 0,783 \\
Habilidade domésticas(HD) & 4 & 56,329 & 0,703 & 0,738 \\
Gestão do lixo (NL) & 2 & 68,259 & 0,826 & - \\
Predisposição (PRED) & 4 & 74,903 & 0,858 & 0,887 \\
\hline
\end{tabular}

Fonte: Dados da Pesquisa (2019).

\subsection{Análise das medidas descritivas}

A segunda etapa de análise dos dados (Tabela 2) consistiu em verificar as medidas descritivas dos construtos, em seguida foi observada a consistência psicométrica das escalas. Levando em conta que a escala para a medição dos construtos foi de 1 a 10, observa-se que de acordo com a média e mediana, os construtos "rotina de compras", "planejamento de compras" e "gestão do lixo" apresentaram o nível de respostas predominantemente intermediários com relação ao grau de concordância. Os construtos "convicções pessoais", "reações emocionais", "habilidades domésticas" e "predisposição" apresentaram níveis elevados de concordância. 
Tabela 2 - Medidas descritivas dos construtos

\begin{tabular}{l|c|c|c|c|c}
\hline \multicolumn{1}{c|}{ Construto } & Média & Mediana & Desvio-padrão & Assimetria & Curtose \\
\hline Convicções pessoais & 8,21 & 9,00 & 1,95 & $-1,108$ & 0,406 \\
Reações emocionais & 7,87 & 8,20 & 1,93 & $-0,883$ & 0,171 \\
Rotina de compras & 4,73 & 4,60 & 2,14 & 0,158 & $-0,651$ \\
Planejamento de compras & 5,93 & 6,00 & 2,22 & $-0,053$ & $-0,590$ \\
Habilidade domésticas & 7,47 & 7,75 & 1,97 & $-0,454$ & $-0,687$ \\
Gestão do lixo & 4,96 & 5,00 & 2,31 & 0,045 & $-0,651$ \\
Predisposição & 8,50 & 9,25 & 1,80 & $-1,117$ & 0,329 \\
\hline
\end{tabular}

Fonte: Dados da Pesquisa (2019)

Quanto a mediana, especificamente, verificou-se que existe simetria em relação à média, não apresentando grande disparidade quando se compara os valores de ambas. Para o desvio padrão estima-se que as variáveis que apresentaram valores até 2 indicam baixa dispersão, entre 2 e 3 eram indicativos de dispersão moderada (Hair, et al, 2005). De acordo com a definição dos parâmetros, as medidas dos construtos "reações emocionais", "rotinas de compra", "planejamento de compras" e "gestão do lixo" indicam que há moderada convergência de posições dos respondentes em torno das médias, sendo destacada a maior dispersão de resposta no construto "gestão do lixo" $(2,31)$ e a menor no construto "predisposição" $(1,80)$.

Quanto às medidas de assimetria e curtose, é possível observar que, as variáveis apresentaram valores entre -1 e +1 , o que, na extração do SPSS, sinaliza que as variáveis podem ser tratadas como normalmente distribuídas, as demais variáveis comportam-se fora do padrão da normalidade, demostrando valores sutilmente elevados quando comparados aos valores de referência.

\subsection{Análise sobre a predisposição para a redução do desperdício alimentar}

Para analisar a predisposição para a redução do desperdício alimentar (doravante "pred isposição"), uma série de técnicas foi efetuada em sequência. O intuito foi explorar potenciais preditores, agrupamentos e fatores moderadores do modelo em estudo. A primeira etapa consistiu em verificar se as variáveis categóricas apresentam, em média, diferentes percepções por grupos. Os resultados a seguir representam aqueles que se mostraram significantes para a pesquisa. Isto é, o que ficou de fora não apresentou diferenças significativas nos testes realizados.

Dito isso, o teste $t$ para amostras independentes foi realizado para verificar se homens e mulheres diferem no escore médio indicado sobre a "predisposição". O resultado sugeriu que as mulheres apresentaram maior predisposição em relação aos homens $\left(\mu_{\mathrm{m}}=9,03 ; \mu_{\mathrm{h}}=5,27 ; \mathrm{t}=7,53 ; \mathrm{p}<0,001\right)$. Ou seja, é possível que a intenção comportamental seja moderada para esses dois estratos demográficos, o que será verificado posteriormente. 
Em seguida, realizou-se análise de variância para verificar se a quantidade de vezes que o respondente indicou cozinhar a própria refeição estava associado a maiores escores médios de "predisposição". O resultado sugeriu que os indivíduos que cozinham a própria refeição apenas um dia por semana possuem menor "predisposição" do que aqueles que cozinham entre 2 e 4 dias e mais de 5 dias por semana $\left(\mu_{1 \mathrm{x}}=7,34 ; \mu_{2 \mathrm{a} 4}=8,77\right.$; $\left.\mu_{5 x}=8,98 ; p<0,001\right)$. Uma comparação do valor da média dos três grupos, sugere uma possível distinção entre o primeiro grupo e os demais. Ou seja, os dois últimos grupos são mais semelhantes no escore de "predisposição".

Na sequência, verificou-se que o nível de escolaridade também contribui para distinguir grupos nesse contexto. Isso foi verificado por meio do teste não paramétrico de Kruskal-Wallis. O resultado sugeriu que indivíduos com nível fundamental de escolaridade apresentaram escore médio menor comparativamente aos dos demais grupos em termos de "predisposição" $\left(\mu_{\mathrm{EF}}=5,90 ; \mu_{\mathrm{EM}}=8,96 ; \mu_{\mathrm{ES}}=8,84 ; \mu_{\mathrm{EPG}}=8,94\right.$; $\left.\chi^{2}=116,27 ; \mathrm{gl}=3 ; \mathrm{p}<0,001\right)$. Aqui também se percebe semelhanças entre os três grupos com maior escolaridade em contraste com os indivíduos com formação de nível fund amental.

Após essas verificações, procedeu-se com a análise do coeficiente de correlação de Pearson para averiguar a associação das variáveis independentes (vide hipóteses de pesquisa) com a "predisposição". Uma análise da matriz de correlação sugeriu maior associação da "pred isposição" com "convicções pessoais" ( $r=0,642 ; p<0,001)$, "reações emocionais" ( $r=0,653 ; p<0,001)$ e "habilidades domésticas" $(r=0,499 ; p<0,001)$. As demais variáveis tiveram menor associação, tod as com valor inferior à 0,3 .

Em seguida, procedeu-se à estimação de modelo de regressão para verificar a influência das variáveis independentes sobre a variação da "predisposição". Os coeficientes foram estimados por meio dos mínimos quadrados ordinários e com método stepwise. Após três iterações, o modelo final incluiu as variáveis "reações emocionais" $(\beta=0,340 ; p<0,001)$, "convicções pessoais" $(\beta=0,338 ; p<0,001)$ e "habilidades domésticas" $(\beta=0,204 ; p<0,001)$ como preditoras da "predisposição". O nível de explicação do modelo obtido foi de 55,8\%. Uma análise dos valores de assimetria $(-0,169)$ e curtose $(0,882)$ dos resíduos do modelo (estimadores do erro), sugeriram adequação à distribuição normal.

Tabela 3 - Modelo de regressão linear estimado

\begin{tabular}{|c|c|c|c|c|c|c|}
\hline \multirow{2}{*}{ Preditores } & \multirow{2}{*}{$\boldsymbol{\beta}$} & \multicolumn{2}{|c|}{ Intervalo de confiança $(95 \%)$} & \multirow[b]{2}{*}{$\mathbf{t}$} & \multirow{2}{*}{ p-valor } & \multirow{2}{*}{ Tolerância } \\
\hline & & $\mathbf{L I}$ & LS & & & \\
\hline Intercepto & 1,601 & 1,061 & 2,142 & 5,820 & 0,000 & - \\
\hline $\begin{array}{l}\text { Reações } \\
\text { emocionais (RE) }\end{array}$ & 0,340 & 0,272 & 0,407 & 9,886 & 0,000 & 0,614 \\
\hline $\begin{array}{l}\text { Convicções } \\
\text { pessoais (CP) }\end{array}$ & 0,338 & 0,268 & 0,408 & 9,475 & 0,000 & 0,625 \\
\hline $\begin{array}{l}\text { Habilidades } \\
\text { domésticas (HD) }\end{array}$ & 0,204 & 0,140 & 0,269 & 6,189 & 0,000 & 0,779 \\
\hline \multicolumn{7}{|l|}{ Medidas de ajuste } \\
\hline \multicolumn{3}{|c|}{$\begin{array}{l}\text { Estatística } \mathrm{F}(8,228 \mathrm{gl}) \text { (p-valor) } \\
\mathrm{R}^{2} \text { ajustado } \\
\text { Assimetria e curtose dos resíduos do modelo }\end{array}$} & \multicolumn{2}{|c|}{$\begin{array}{l}32,889(\mathrm{p}=0,000) \\
0,558 \\
-0,169 ; 0,882\end{array}$} & & \\
\hline
\end{tabular}

Fonte: Dados da pesquisa (2019). 
A última análise realizada consistiu na verificação de possíveis agrupamentos com base nas variáveis do modelo estimado na Tabela 3 ("predisposição", "reações emocionais", "convicções pessoais" e "habilidades domésticas"). Para isso, procedeu-se com a análise de conglomerados não-hierárquicos com base em dois grupos. Os resultados obtidos indicaram uma diferenciação de perfis com base nas variáveis preditoras. $\mathrm{O}$ grupo 1 teve escores médios superiores $\left(\mu_{\mathrm{CP}}=9,14, \mu_{\mathrm{RE}}=8,84, \mu_{\mathrm{HD}}=7,73\right)$ comparativamente aos do grupo $2\left(\mu_{\mathrm{CP}}=6,07, \mu_{\mathrm{RE}}=5,75, \mu_{\mathrm{HD}}=5,81\right)$. Todas as três variáveis contribuíram para distinção entre os agrupamentos $(\mathrm{p}<0,001)$. Com isso, decidiu-se nomear o primeiro agrupamento como "Predispostos a redução do desperdício alimentar" e o segundo como "Não-predispostos a redução do desperdício alimentar".

O grupo "predispostos a redução do desperdício alimentar" ficou composto majoritariamente por mulheres (62,7\%), indivíduos entre 21 e 35 anos (60,2\%), pessoas com ensino superior $(49,6 \%)$, respondentes que trabalham o dia inteiro $(38,8 \%)$ ou estudantes $(31,9 \%)$, e aqueles que cozinham a sua própria refeição mais de uma vez por semana (85,3\%). Já o grupo de "não-predispostos a redução do desperdício alimentar" foi composto, em sua maioria, por homens (70,6\%), indivíduos com mais de 50 anos de idade $(30,8 \%)$, pessoas com nível de escolaridade fundamental $(42,7 \%)$, respondentes aposentados $(35 \%)$ e casos que cozinham sua própria comida apenas um dia na semana $(49 \%)$.

Tais resultados apontam para as diversas possibilidades de abordagens que podem direcionar as ações de marketing social. Os apontamentos acerca das hipóteses propostas no estudo e os demais resultados que emergiram na análise da predisposição para a redução do desperdício alimentar serão discutidos a seguir.

\section{DISCUSSÃO DOS RESULTADOS}

Com base nos resultados obtidos, algumas das hipóteses que nortearam o estudo foram rejeitadas, uma vez que vários dos condicionantes não demonstraram impacto na pesquisa empreendida, contrariando resultados de outros estudos.

No que se refere a $\mathrm{H} 1$, os resultados apontaram para a sua não rejeição, confirmando os achados das pesquisas de Stancu, Haugaard \& Lähteenmäki (2015) e Visschers, Wickli \& Siegrist (2016), os quais reconheceram que a sensibilização em torno de comportamentos pró-ambientais repercute positivamente na redução do desperdício alimentar. Ou seja, as campanhas de marketing social voltadas para o desperdício alimentar devem iniciar suas propostas a partir de uma discussão sobre a moralidade de tal prática, ressaltando os significados culturais, históricos e emocionais atribuídos aos alimentos na sociedade. A hipótese $\mathrm{H} 2$ obteve como resultado a sua não rejeição, de modo que as reações emocionais relativas ao sentimento de culpa por desperdiçar alimentos se confirmaram como preditores para a adoção da redução do desperdício.

A hipótese $\mathrm{H} 3$ foi rejeitada, assim se compreendeu que a rotina de compras dos indivíduos não influencia a predisposição em reduzir o desperdício alimentar. Tal resultado contrapõe diversos estudos Porpino et al. (2015); Visschers; Wickli, \& Siegrist (2016) e Richter (2017), já que a organização alimentar se traduz em idas aos supermercados e compras mais sistematizadas. A hipótese H4 resultou em não rejeição, embora a rotina de compras não tenha se confirmado como um preditor, as habilidades domésticas dos indivíduos na cozinha mostraram influência na redução do desperdício 
alimentar. Já a hipótese H5 foi rejeitada, resultando na ausência de influência do planejamento alimentar para a redução do desperdício. A negação da expectativa de influência das hipóteses H3 e H5 são explicadas por aspectos culturais, o que sugere a avaliação em outros estudos.

Em reflexão aos achados das hipóteses supracitadas, é possível compreender que o fator preponderante para a redução do desperdício reside na habilidade do indivíduo de manipular os alimentar e saber cozinhar. Isto direciona as atividades de marketing social para um caminho de promoção de maior autonomia para os indivíduos, tornandoos aptos a manipular utensílios de cozinha, fracionar as refeições, criar receitas com as sobras e também identificar as partes comestíveis de cada alimento. Analisando a rejeição da rotina de compras e do planejamento alimentar, torna-se necessário compreender quais alimentos são rotineiramente adquiridos pelos consumidores e como esses alimentos são consumidos em sua residência. Os resultados descritivos desses construtos apontaram para um moderado nível de concordância com as afirmativas, de forma que o desajuste na rotina de compras e no planejamento não influencia na pred isposição dos sujeitos em reduzir o desperdício de alimentos.

Por último, a hipótese $\mathrm{H} 6$ foi rejeitada. O entendimento de que a percepção do sujeito sobre a gestão do seu lixo não influencia a predisposição para a redução do desperdício alimentar expõe um claro descompasso. É evidente que não há consciência sobre as diferenças entre lixo e desperdício, há ainda um baixo nível de concordância relativo à produção do lixo alimentar impactar o meio ambiente. Embora tal construto não tenha sido preditor da redução do desperdício alimentar, as ações de marketing social voltadas para a temática devem esclarecer os impactos sociais, econômicos e ambientais da gestão adequada dos resíduos sólidos das cidades. É preciso vincular políticas de reciclagem com ações de redução do desperdício alimentar, na medida em que o reaproveitamento de alimentos para novas receitas ou compostagem se relaciona fortemente com a redução do consumo de produtos de uso único, como os descartáveis.

No geral, as hipóteses não rejeitadas conseguem explicar em 55,8\% a predisposição em reduzir o desperdício alimentar. Indo além do debate promovido pelas hipóteses, os resultados apontam ainda para maior predisposição das mulheres em ad otar ações de desperdício alimentar. O que configura um achado importante, tendo em vista que um dos elementos fundamentais para construção de intervenções de marketing social é justamente a segmentação de grupos específicos, para melhor direcionar os esforços para promoção da mudança de comportamento (Chau et al., 2018). Nesse sentido, as mulheres configuram um grupo em potencial, considerando que apresentam maior predisposição em adotar práticas de combate ao desperdício.

Um reforço a esse entendimento é ad vindo do estudo de Koivupuro et al. (2012) que buscou entender as influências sociodemográficas na quantidade de alimentos desperdiçados por famílias finlandesas, em que se identificou que a quantidade de alimentos desperdiçados era maior entre as famílias em que a mulher era a principal responsável pelas compras se comparado aos domicílios em que apenas o homem ou os dois cônjuges eram responsáveis. Já no estudo de Cantaragiu (2019), constatou-se que as mulheres tendem a se preocupar mais com os impactos negativos do desperdício alimentar, seja relacionado a equidade social ou no orçamento familiar se comparada ao nível de preocupação dos homens. Embora, como também constatado no estudo de Koivupuro et al. (2012), as mulheres demonstrem comportamentos de aquisição e preparação de alimentos que podem resultar em desperdício de alimentos em maior grau que os homens.

Tais informações indicam que as mulheres constituem um grupo alvo em potencial para intervenções de combate ao desperdício, pois carecem de maiores 
informações quanto ao planejamento de compras e um comportamento mais adequado nesse sentido aumentaria a probabilidade de reduções significativas do desperdício de alimentos. Considerando que mesmo o presente estudo constatando que a compra não interfere na predisposição para redução do desperdício alimentar, o elemento compra exerce influência direta no contexto do desperdício alimentar doméstico conforme Di Talia (2019), principalmente se realizada de forma improvisada.

Além disso, as mulheres são mais preocupadas com os impactos negativos ocasionados pelo desperdício e mais predispostas a adotar ações para redução do desperdício. Para tanto, é possível observar diferenças significativas entre homens e mulheres no que tange o contexto do desperdício alimentar, de modo que para construção de intervenções de marketing social que objetivem atender os dois públicos são necessários elementos específicos para cada gênero.

Outro apontamento dos resultados diz respeito aos indivíduos que cozinham apenas um dia na semana apresentarem menor predisposição para redução do desperdício alimentar. Corroborando em certa medida com os achados de Chen (2019) que apontou que a frequência de preparação de comida caseira influencia significativamente o comportamento de prevenção de desperdício de alimentos dos consumidores. Justificando que o envolvimento com alimentação, nos diversos processos, aquisição, planejamento, preparação e descarte de alimentos podem interferir nesses aspectos, pois o envolvimento emocional pode refletir nos hábitos alimentares e corroborar para redução do desperdício. De maneira que Chen (2019) defende que os programas voltados a redução do desperdício alimentar devem incentivar a preparação de comida caseira, educando e treinando os indivíduos nesses aspectos, pois além de permitir que as pessoas reconheçam o valor dos recursos alimentares, também pode capacitar as pessoas a minimizarem o consumo doméstico.

Ademais, os sujeitos que possuem o nível fundamental de escolaridade apresentaram resultados inferiores quando comparados aos indivíduos com mais instrução. Esse achado pode ser explicado pelos resultados da Di Talia (2019), na medida que se identificou que as pessoas com maior grau de instrução dedicam mais atenção ao desperdício de alimentos. Desse modo podem ser mais conscientes a respeito dos danos ocasionados pelo desperdício alimentar.

\section{CONSIDERAÇÕES FINAIS}

Os estudos sobre desperdício alimentar sob a perspectiva de marketing social convergem a partir da contribuição para o planejamento das instituições públicas e organizações sociais que atuam em torno de questões alimentares. Este estudo objetivou contribuir com esse contexto, na medida em que propôs uma análise sobre os fatores que influenciam a predisposição para a redução do desperdício alimentar, em uma perspectiva clássica das análises de marketing social.

Para isto, foi realizado um levantamento empírico, cujos resultados indicaram que três aspectos são influenciadores da predisposição das pessoas em adotar ações de redução de desperdício alimentar, quais sejam: as convicções emocionais, as reações emocionais e as habilidades domésticas. De maneira geral, este artigo propiciou um entendimento sobre o contexto geral do desperdício alimentar, ao passo que pode servir de referência para outras pesquisas que abordem tal relação em marketing social.

De um ponto de vista prático, os resultados sinalizaram variáveis relevantes para o planejamento de ações de redução do desperdício, indicando que os gestores públicos e os profissionais de marketing social devem adotar apelos morais e éticos nas campanhas, ao mesmo tempo em que oferta e capacita dos indivíduos no manuseio dos 
alimentos. Isto pode ocorrer em escolas, universidades e outras repartições de ensino. Ademais, é preciso buscar a segmentação como uma estratégia eficaz para a definição dos estímulos adequados a cada público-alvo.

Adicionalmente, em termos teóricos, as análises estatísticas não são suficientes para uma compreensão mais plena do comportamento, de modo que é recomendado que estudos qualitativos aprofundem o entendimento sobre as hipóteses rejeitadas, uma vez que outros estudos ressaltaram sua relevância. Por fim, pelo fato de a amostra ter sido acessada por conveniência e ter um perfil com nível de instrução elevado, tais resultados não podem ser generalizados; logo, recomendam-se estudos que empreguem procedimentos de amostragem mais rigorosos.

\section{REFERENCIAS}

Andreasen, A. R. (1994). Social marketing: Its definition and domain. Journal of public policy \& marketing, 13(1), 108-114.

Bell, D. R., Corsten, D., \& Knox, G. (2011). From point of purchase to path to purchase: How preshopping factors drive unplanned buying. Journal of Marketing, 75(1), 31-45.

Benyam, A., Kinnear, S., \& Rolfe, J. (2018). Integrating community perspectives into domestic food waste prevention and diversion policies. Resources, Conservation and Recycling, 134, 174-183.

Brook, L. (2007). Food behaviour consumer research-findings from the quantitative survey. Briefing Paper. UK: WRAP. Project code: RWM005-001.

Caisan, C. I. D. S. A. Nutricional-2018. Estratégia Intersetorial para a Redução de Perdas e Desperdício de Alimentos no Brasil. Brasil: Ministério do Desenvolvimento Social MDS.

Cantaragiu, R. (2019). The Impact of Gender on Food Waste at the Consumer Level. Studia Universitatis „,Vasile Goldis” Arad-Economics Series, 29(4), 41-57.

Cantaragiu, R. (2019). The impact of gender on food waste at the consumer level. Studia Universitatis Vasile Goldiş, Arad-Seria Ştiinţe Economice, 29(4), 41-57.

Carins, J. E., Rundle-Thiele, S. R., \& Parkinson, J. E. (2017). Delivering healthy food choice: A dual-process model enquiry. Social marketing quarterly, 23(3), 266-283.

Chan, K. Y., Orr, L., Fahey, D., \& Dorahy, C. G. (2011). Agronomic and economic benefits of garden organics compost in vegetable production. Compost science \& utilization, 19(2), 97-104.

Chau, J. Y., McGill, B., Thomas, M. M., Carroll, T. E., Bellew, W., Bauman, A., \& Grunseit, A. C. (2018). Is this health campaign really social marketing? A checklist to help you decide. Health Promotion Journal of Australia, 29(1), 79-83. 
Chau, J. Y., McGill, B., Thomas, M. M., Carroll, T. E., Bellew, W., Bauman, A., \& Grunseit, A. C. (2018). Is this health campaign really social marketing? A checklist to help you decide. Health Promotion Journal of Australia, 29(1), 79-83.

Chen, H. S. (2019). Environmental Concerns and Food Consumption: What Drives Consumers' Actions to Reduce Food Waste?. Journal of International Food \& Agribusiness Marketing, 31(3), 273-292.

Costa, F. J. (2011) Mensuração e Desenvolvimento de Escalas: Aplicações em administração. Rio de Janeiro: Editora Ciência Moderna.

Cox, E. P. (1980). The Optimal Number of Response Alternatives for a Scale: A Review. Journal of Marketing Research, 17(4), 407-422.

Di Talia, E., Simeone, M., \& Scarpato, D. (2019). Consumer behaviour types in household food waste. Journal of cleaner production, 214, 166-172.

Diaz-Ruiz, R., Costa-Font, M., \& Gil, J. M. (2018). Moving ahead from food-related behaviours: an alternative approach to understand household food waste generation. Journal of cleaner production, 172, 1140-1151.

Dibb, S. (2014). Up, up and away: social marketing breaks free. Journal of Marketing Management, 30(11-12), 1159-1185.

FAO. (2019). The State of Food and Agriculture 2019. Moving Forward on Food Loss and Waste Reduction.

Firestone, R., Rowe, C. J., Modi, S. N., \& Sievers, D. (2017). The effectiveness of social marketing in global health: A systematic review. Health policy and planning, $32(1), 110-124$.

Gjersing L, Caplehorn JR, Clausen T. Cross-cultural adaptation of research instruments: Language, setting, time and statistical considerations. BMC Med Res Methodol 2010; 10:13.

Graham-Rowe, E., Jessop, D. C., \& Sparks, P. (2014). Identifying motivations and barriers to minimising household food waste. Resources, conservation and recycling, $84,15-23$.

Graham-Rowe, E., Jessop, D. C., \& Sparks, P. (2015). Predicting household food waste reduction using an extended theory of planned behaviour. Resources, Conservation and Recycling, 101, 194-202.

Hair, J. F., Black, W. C., Babin, B. J., Anderson, R. E., \& Tatham, R. L. (2005). Multivariate Data Analysis (6th ed.). Prentice-Hall International.

Hair, J. R. JF et al.(2005). Análise multivariada de dados. Trad. Adonai Schlup Sant'Anna e Anselmo Chaves Neto. 
Halloran, A., Clement, J., Kornum, N., Bucatariu, C., \& Magid, J. (2014). Addressing food waste reduction in Denmark. Food Policy, 49, 294-301.

Hebrok, M., \& Heidenstrøm, N. (2019). Contextualising food waste preventionDecisive moments within everyday practices. Journal of cleaner production, 210, 14351448 .

Hodgkins, S., Rundle-Thiele, S., Knox, K., \& Kim, J. (2019). Utilising stakeholder theory for social marketing process evaluation in a food waste context. Journal of Social Marketing.

Jager, J., Putnick, D. L., \& Bornstein, M. H. (2017). More than Just Convenient: The Scientific Merits of Homogeneous Convenience Samples. Monographs of the Society for Research in Child Development, 82(2), 13-30.

Khalid, S., Naseer, A., Shahid, M., Shah, G. M., Ullah, M. I., Waqar, A., ... \& Rehman, F. (2019). Assessment of nutritional loss with food waste and factors governing this waste at household level in Pakistan. Journal of cleaner production, 206, 1015-1024.

Kim, J., Rundle-Thiele, S., \& Knox, K. (2019). Systematic literature review of best practice in food waste reduction programs. Journal of Social Marketing.

Kim, J. Rundle-thiele, S. Knox, K. Systematic literature review of best practice in food waste reduction programs. Journal of Social Marketing, 2019.

Koivupuro, H. K., Hartikainen, H., Silvennoinen, K., Katajajuuri, J. M., Heikintalo, N., Reinikainen, A., \& Jalkanen, L. (2012). Influence of socio-demographical, behavioural and attitudinal factors on the amount of avoidable food waste generated in Finnish households. International journal of consumer studies, 36(2), 183-191.

Lattin, J., Carroll, J. D., \& Green, P. E. (2011). Análise de dados multivariados. São Paulo: Cengage Learning, 475.

Lundie, S., \& Peters, G. M. (2005). Life cycle assessment of food waste management options. Journal of cleaner production, 13(3), 275-286.

Manion, M. (1994). Survey Research in the Study of Contemporary China: Learning from Local Samples. The China Quarterly, 139, 741.

Mirosa, M., Munro, H., Mangan-Walker, E., \& Pearson, D. (2016). Reducing waste of food left on plates. British Food Journal.

Mondéjar-Jiménez, J. A., Ferrari, G., Secondi, L., \& Principato, L. (2016). From the table to waste: An exploratory study on behaviour towards food waste of Spanish and Italian youths. Journal of Cleaner Production, 138, 8-18.

Närvänen, E., Mattila, M., \& Mesiranta, N. (2019). Consumer-citizens as leaders of change: Case food waste. Leading change in a complex world: Transdisciplinary perspectives. 
Närvänen, E., Mesiranta, N., Mattila, M., \& Heikkinen, A. (2020). Introduction: A Framework for Managing Food Waste. In Food Waste Management (pp. 1-24). Palgrave Macmillan, Cham.

Östergren, K., Gustavsson, J., Bos-Brouwers, H., Timmermans, T., Hansen, O. J., Møller, H., ... \& Easteal, S. (2014). FUSIONS definitional framework for food waste. Wageningen: FUSIONS Project.

Parizeau, K., von Massow, M., \& Martin, R. (2015). Household-level dynamics of food waste production and related beliefs, attitudes, and behaviours in Guelph, Ontario. Waste management, 35, 207-217.

Pellegrini, G., Sillani, S., Gregori, M., \& Spada, A. (2019). Household food waste reduction: Italian consumers' analysis for improving food management. British Food Journal.

Porpino, G., Parente, J., \& Wansink, B. (2015). Food waste paradox: antecedents of food disposal in low income households. International journal of consumer studies, $39(6), 619-629$.

Porpino, G.; Lourenço, C. E.; Araújo, C.M.; Bastos, A. (2018). Intercâmbio Brasil União Europeia sobre desperdício de alimentos. Relatório final de pesquisa. Brasília: Diálogos Setoriais União Europeia - Brasil. Disponível em: < http:// www.sectordialogues.org/publicacao.

Quested, T. E., Marsh, E., Stunell, D., \& Parry, A. D. (2013). Spaghetti soup: The complex world of food waste behaviours. Resources, Conservation and Recycling, 79, 43-51.

Ramirez, A. S., Rios, L. K. D., Valdez, Z., Estrada, E., \& Ruiz, A. (2017). Bringing produce to the people: implementing a social marketing food access intervention in rural food deserts. Journal of nutrition education and behavior, 49(2), 166-174.

Reisch, L., Eberle, U., \& Lorek, S. (2013). Sustainable food consumption: an overview of contemporary issues and policies. Sustainability: Science, Practice and Policy, 9(2), $7-25$.

Richter, B. (2017). Knowledge and perception of food waste among German consumers. Journal of Cleaner Production, 166, 641-648.

Richter, B. (2017). Knowledge and perception of food waste among German consumers. Journal of Cleaner Production, 166, 641-648.

Robinson, J. P., Shaver, P. R., Wrightsman, L. S., \& Andrews, F. M. (Orgs.). (1991). Measures of personality and social psychological attitudes. Academic Press.

Rundle-Thiele, S., David, P., Willmott, T., Pang, B., Eagle, L., \& Hay, R. (2019). Social marketing theory development goals: an agenda to drive change. Journal of Marketing Management, 35(1-2), 160-181. 
Santini, C., \& Cavicchi, A. (2014). The adaptive change of the Italian Food Bank found ation: a case study. British Food Journal, 116(9), 1446-1459.

Silchenko, K., Simonetti, F., \& Gistri, G. (2019). The Second Life of Food: When Social Marketing Bridges Solidarity and Waste Prevention. In Social Marketing in Action (pp. 111-126). Springer, Cham.

Soares, A. B. (2018). Perdas e desperdícios de alimentos no Brasil: efeitos da redução na colheita e pós-colheita.

Soraghan, C., Thomson, E., \& Ensor, J. (2016). Using food labels to evaluate the practice of nudging in a social marketing context. Social Business, 6(3), 249-265.

Southerton, D., \& Yates, L. (2015). Exploring food waste through the lens of social practice theories. In Waste management and sustainable consumption (Vol. 133, No. 149, pp. 133-149). ROUTLEDGE in association with GSE Research.

Stancu, V., Haugaard, P., \& Lähteenmäki, L. (2016). Determinants of consumer food waste behaviour: Two routes to food waste. Appetite, 96, 7-17.

Stefan, V., van Herpen, E., Tudoran, A. A., \& Lähteenmäki, L. (2013). Avoiding food waste by Romanian consumers: The importance of planning and shopping routines. Food Quality and Preference, 28(1), 375-381.

Tobey, L. N., Koenig, H. F., Brown, N. A., \& Manore, M. M. (2016). Reaching lowincome mothers to improve family fruit and vegetable intake: Food Hero social marketing campaign — research steps, development and testing. Nutrients, 8(9), 562.

Visschers, V. H., Wickli, N., \& Siegrist, M. (2016). Sorting out food waste behaviour: A survey on the motivators and barriers of self-reported amounts of food waste in households. Journal of Environmental Psychology, 45, 66-78.

Watson, M., \& Meah, A. (2012). Food, waste and safety: Negotiating conflicting social anxieties into the practices of domestic provisioning. The Sociological Review, 60, 102120.

Williams, H., Wikström, F., Wetter-Edman, K., \& Kristensson, P. (2018). Decisions on recycling or waste: How packaging functions affect the fate of used packaging in selected Swedish households. Sustainability, 10(12), 4794.

Worsley, A., Wang, W. C., \& Burton, M. (2015). Food concerns and support for environmental food policies and purchasing. Appetite, 91, 48-55. 